\title{
The relationship among periodontal condition, serum lipid, and electrocardiographic abnormalities in the elderly: A prospective cohort study
}

\author{
Lisdrianto Hanindriyo $^{1,2}$, Akihiro Yoshihara ${ }^{3}$, Toshinobu Hirotomi ${ }^{1}$, Hideo Miyazaki ${ }^{1}$ \\ ${ }^{1}$ Division of Preventive Dentistry, Department of Oral Health Science, Graduate School of Medical and Dental Science, Niigata \\ University, Niigata, Japan \\ ${ }^{2}$ Department of Preventive and Community Dentistry, Faculty of Dentistry, Gadjah Mada University, Yogyakarta, Indonesia \\ ${ }^{3}$ Division of Oral Science for Health Promotion, Department of Oral Health and Welfare, Graduate School of Medical and Dental \\ Science, Niigata University, Niigata, Japan \\ Email: lisdrianto_hanindriyo@ugm.ac.id
}

Received 30 September 2013; revised 31 October 2013; accepted 11 November 2013

Copyright (C) 2013 Lisdrianto Hanindriyo et al. This is an open access article distributed under the Creative Commons Attribution License, which permits unrestricted use, distribution, and reproduction in any medium, provided the original work is properly cited.

\begin{abstract}
Periodontal disease is recognized as a risk factor for cardiovascular disease (CVD). Some studies have suggested that serum lipids might play a role in the association between periodontal disease and CVD. However, this hypothesis remains unproven. Present study evaluated the association between periodontal disease and CVD-related parameters including electrocardiographic (ECG) parameters and serum lipid levels in the elderly. This study was a prospective cohort study. A total of 107 subjects (57 males and 50 females) from Niigata City's community-dwelling elderly (all aged 75 years old) who possessed at least 20 teeth and did not exhibit ECG abnormalities at the baseline underwent annual examinations for 5 years. Logistic regression analysis was used to assess the relationship between periodontal condition and the presence of ECG abnormalities, after adjusting for confounding factors including serum lipid levels. Logistic regression analysis showed that the subjects who exhibited a poor periodontal condition at the baseline had a 2.97 times greater risk of suffering from ECG abnormalities than the subjects who displayed a good periodontal condition at the baseline ( $p$ $=0.019)$. Furthermore, the subjects who displayed a poor periodontal condition and an unfavorable serum lipid profile at the baseline showed a significantly higher frequency of ECG abnormalities (63.6\%) than those who exhibited a healthy periodontal condition and a favorable serum lipid profile $(21.6 \%, p=$ 0.008 ) as well as those who displayed a healthy periodontal condition and an unfavorable serum lipid pro-
\end{abstract}

file $(21.4 \%, p=0.010)$. As a conclusion, periodontal condition could be a marker of CVD, even in the healthy elderly.

Keywords: Periodontal Condition; Serum Lipid; Cardiovascular Disease; Elderly People

\section{INTRODUCTION}

It has been determined that poor oral health can have a profound effect on general health, and several oral diseases are known to be associated with chronic diseases [1]. Besides the fact that periodontal disease is one of two major dental diseases that display high prevalence rates worldwide [2], the World Health Organization (WHO) has also found that periodontal disease shares some common risk factors with systemic diseases such as cardiovascular disease (CVD) [3].

Previous studies have suggested that serum lipids might play a role in the association between periodontal disease and CVD, particularly in elderly patients. While a reduced high-density lipoprotein cholesterol (HDL-C) level is a well-recognized risk factor for CVD and ischemic stroke [4,5]. Izumi et al. [6] found that a higher total cholesterol (TC) level is associated with a lower prevalence of periodontitis in non-smoking elderly people. Moreover, two cross-sectional studies have been conducted in Japan to assess the associations between blood chemical parameters and periodontal status, and both studies indicated that elevated HDL-C levels are correlated with a reduced incidence of periodontal disease $[7,8]$.

On the other hand, other studies failed to identify any 
association between periodontal disease and CVD. HolmPedersen et al. [9] investigated the correlations among dental caries, periodontal disease, and cardiac arrhythmia in community-dwelling elderly individuals who were aged 80 or older and showed that there was no association between periodontal disease and arrhythmia. In line with this result, a case-control study by Sridhar et al. [10] found that coronary heart disease did not exacerbate the destruction of periodontal tissue.

The inconsistencies between these studies might have been due to differences in study design, the adjustments made for confounding variables, and other methodological details. Some previous studies used a case-control design, which is known to introduce various types of bias [11], while other studies employed a cross-sectional design, which made it impossible to assess the direction of the relationship between the assumed cause and effect [12]. Furthermore, some previous studies might have inadequately controlled for confounding factors such as gender, age, smoking status/history, and body mass index (BMI).

Therefore, the purpose of this study was to evaluate the association between periodontal disease and CVDrelated parameters including electrocardiographic (ECG) parameters and serum lipid levels in the elderly using a prospective cohort study design. The result of this study was considered to be important in giving more insights for the elucidation of the correlation between oral and general health.

\section{MATERIALS AND METHODS}

\subsection{Subjects}

The subjects for this study was drawn from the Niigata Elderly Study, which was a community-based prospective study that aimed to assess the relationship between oral health and general health in the elderly. Invitations were sent to all residents $(n=4542)$ who were born in 1927 based on the Niigata City's resident registry. The invitations also informed the recipients of the purpose of the study. As a result, 81.4\% $(n=3695)$ of the invitees agreed to participate in the study. Considering the availability of resources, appointments for examinations could only be arranged for 600 individuals. The final study subjects $(n=600)$ were randomly selected so that they included approximately equal numbers of men (306) and women (294). We assessed the general condition of the subjects using the Tokyo Metropolitan Institute of Gerontology Index (TMIG-Index) of Competence subscale questionnaires. All of the subjects were Japanese, in good general health, and did not require special care for their daily activities. The subjects' mean score on the TMIG-Index subscales was $11.9 \pm 1.4$, and the results of this assessment were indicative of a high level of competence among the study participants. The 600 subjects were invited for annual examinations including oral and general physical examinations from 1998 to 2008. All of the subjects agreed and signed informed consent forms regarding the examination protocols. Of the 600 subjects, 312 participated in all of the annual examinations from 2003 to 2008. An age of 75 years in 2003 was set as the baseline for the present study since a recent review showed that approximately $50 \%$ of patients with dysrhythmia, especially atrial fibrillation, were aged 75 years or older [13]. One hundred and seven (57 males and 50 females) of the 312 subjects were selected for the present study because they had at least 20 teeth and did not display any atherosclerosis-related ECG abnormalities at the baseline which was considered as the inclusion criteria for this study. All of the subjects were examined at local community centers in Niigata City. The ethics committee of the Niigata University School of Dentistry approved this study and protected the subjects' rights.

\subsection{Oral Examinations}

The periodontal examinations were carried out by four trained dentists under sufficient illumination with artificial light. Periodontal conditions was assessed using dental mirrors and a specially designed constant-pressure periodontal probe (Vivacare TPS Probe $\AA$; Schaan, Liechtenstein), which was applied at a probing force of $20 \mathrm{~g}$. The probing depth (PD) and clinical attachment level (CAL) at six sites (mesiobuccal, mid-buccal, distobuccal, mesiolingual/palatal, mid-lingual/palatal, and distolingual/palatal) were recorded for all teeth, includeing the third molars, and rounded to the nearest millimeter. The examiners were calibrated before and during the survey, and inter-examiner reliability was assessed. According to replicated examinations of 10 patients, the percentage agreement (within $\pm 1 \mathrm{~mm}$ ) ranged from $87.5 \%$ to $100.0 \%$ for PD and $83.3 \%$ to $100.0 \%$ for CAL. In addition, the Kappa value ranged from 0.8 to 1.0 for PD and 0.7 to 1.0 for CAL. The National Health and Nutrition Examination Survey (NHANES) III [14] found that about $30.0 \%$ of 70 - to 80 -year-old subjects had moderate to advanced periodontitis. Accordingly, we used the 30th percentile to indicate a poor periodontal condition. Therefore, each subject's periodontal condition was classified according to whether their mean PD was $\geq 1.8 \mathrm{~mm}$ or $<1.8 \mathrm{~mm}$ and whether their mean CAL was $\geq 2.5 \mathrm{~mm}$ or $<2.5 \mathrm{~mm}$ [15]. Subjects with a mean PD of $\geq 1.8 \mathrm{~mm}$ and a mean CAL of $\geq 2.5 \mathrm{~mm}$ were considered to exhibit a poor periodontal condition. The mean PD was $2.05 \mathrm{~mm}$, and the mean CAL was $2.98 \mathrm{~mm}$. The number of teeth present was also investigated. 


\subsection{General Examinations}

\subsubsection{Electrocardiography}

A resting 12-lead ECG recording was taken using a standard ECG machine (CardioStar FCP-7411; Fukuda ME Kogyo Co., Tokyo, Japan) equipped with an automated analysis system whilst the subject was in the supine position, in accordance with standard recommendations [16]. The criteria established by Takata et al. [17] were used to diagnose atherosclerosis-related ECG abnormalities. Atrial fibrillation was defined as a markedly irregular R-R interval and the absence of P-waves, with disorganized electrical activity being seen in their place. VPC (Ventricular premature contractions) were defined as an antecedent R-R interval of $\leq 0.8 \times$ the mean $\mathrm{R}-\mathrm{R}$ interval, a subsequent $R-R$ interval of $\geq 1.05 \times$ the mean $\mathrm{R}-\mathrm{R}$ interval, and an abnormal QRS complex. SVPC (Supraventricular premature contractions) were defined as an antecedent R-R interval of $\leq 0.8 \times$ the mean $\mathrm{R}-\mathrm{R}$ interval, a subsequent $R-R$ interval of $\geq 1.05 \times$ the mean $\mathrm{R}-\mathrm{R}$ interval, a normal QRS complex, and no evidence of atrial fibrillation or sinus dysrhythmia. Sinus dysrhythmia was defined as an irregular R-R interval with no other dysrhythmia. Sinus tachycardia was defined as a P-wave rate of greater than 100 beats/min, with no other dysrhythmia. Sinus bradycardia was defined as a P-wave rate of less than 60 beats/min, with no other dysrhythmia. LVH (left ventricular hypertrophy) and ST-segment depression as defined by the Minnesota Code were also evaluated [18]. All of the abovementioned conditions were considered to be abnormal ECG findings. The subjects who displayed any of these ECG abnormalities during a follow-up examination were categorized as "positive", and those that did not were categorized as "negative".

\subsubsection{Blood Pressure, Gender, Smoking History, BMI, and Blood Measurements}

The blood pressure (BP) measurement and blood sampling were performed with the subjects in the supine position. The patients' TC, HDL-C, and low-density lipoprotein cholesterol (LDL-C) levels were determined by a commercial laboratory (BML, Inc., Tokyo, Japan) under non-fasting conditions. Based on the threshold for people in the intermediate-risk group for CVD (possessing 1 - 2 major risk factors for CVD other than LDL-C; age $\geq 45$ for males and $\geq 55$ for females is a major risk factor) suggested by the Japan Atherosclerosis Society [19], subjects with serum LDL-C levels of $<140 \mathrm{mg} / \mathrm{dL}$, serum HDL-C levels of $\geq 40 \mathrm{mg} / \mathrm{dL}$, and serum TC levels of $<220 \mathrm{mg} / \mathrm{dL}$ were considered to display favorable serum lipid profiles, while the subjects that exhibited the opposite findings were considered to display unfavorable serum lipid profiles. Personal interviews were conducted to obtain information regarding the subjects' smoking habits and sex. Anthropometric evaluations including measurements of weight and height were performed to allow BMI calculations to be performed.

\subsection{Statistical Analysis}

Initially, the subjects' characteristics were compared between the subjects with and without ECG abnormalities using the $t$-test for quantitative data and the chi-square test for categorical variables. During this analysis, baseline data regarding the number of remaining teeth; HDLC, LDL-C, and TC levels; and mean PD and CAL were compared.

Furthermore, to analyze the effects of periodontal condition and the serum lipid profile on the presence/absence of ECG abnormalities during the study period, the chi-square test was used to compare the following four groups: the subjects who exhibited a healthy periodontal condition and a favorable serum lipid profile $(\mathrm{n}=37)$; a healthy periodontal condition and an unfavorable serum lipid profile $(n=28)$; a poor periodontal condition and a favorable serum lipid profile $(\mathrm{n}=31)$; and a poor periodontal condition and an unfavorable serum lipid profile $(\mathrm{n}=11)$.

Finally, logistic regression analysis was performed to examine the independent effect of periodontal condition on the presence/absence of ECG abnormalities during the study period. The presence of ECG abnormalities during the study period was used as the dependent variable, while the independent variables were periodontal condition at the baseline and other parameters that were found to be significantly associated with ECG abnormalities in the initial analysis. All calculations and statistical analyses were performed using SPSS version 17. Statistical significance was set at $\alpha=0.05$.

\section{RESULTS}

Table 1 shows the means and distributions of the study variables according to the presence or absence of ECG abnormalities during the study period. The subjects with ECG abnormalities had significantly higher BMI values at the baseline than those without $(23.9 \pm 2.8$ vs $22.6 \pm$ $\left.2.8 \mathrm{~kg} / \mathrm{m}^{2}, p=0.030\right)$. Furthermore, ECG abnormalities were significantly associated with lower LDL and TC levels at the baseline. While only $29.2 \%$ of the subjects who did not exhibit ECG abnormalities displayed a poor periodontal condition, $60.0 \%$ of the subjects with ECG abnormalities displayed a poor periodontal condition $(p=$ 0.002).

As shown in Figure 1, a significantly higher percentage of the subjects who displayed a poor periodontal condition and an unfavorable serum lipid profile at the baseline suffered from ECG abnormalities (63.6\%) 
Table 1. Background characteristics of the subjects according to their ECG status.

\begin{tabular}{|c|c|c|c|}
\hline \multirow{4}{*}{ Subjects' characteristics } & \multicolumn{2}{|c|}{ ECG abnormalities } & \multirow[b]{4}{*}{$p$-value ${ }^{a}$} \\
\hline & Negative & Positive & \\
\hline & $\mathrm{n}=72$ & $\mathbf{n}=35$ & \\
\hline & \multicolumn{2}{|c|}{ Mean \pm SD } & \\
\hline Present teeth at the baseline & $25.1 \pm 2.9$ & $25.4 \pm 3.0$ & 0.641 \\
\hline BMI at the baseline $\left(\mathrm{kg} / \mathrm{m}^{2}\right)$ & $22.6 \pm 2.8$ & $23.9 \pm 2.8$ & 0.030 \\
\hline Mean BMI over 5 years $\left(\mathrm{kg} / \mathrm{m}^{2}\right)$ & $22.1 \pm 2.7$ & $23.2 \pm 2.9$ & 0.060 \\
\hline Systolic BP at the baseline (mmHg) & $129.9 \pm 15.3$ & $133.8 \pm 17.4$ & 0.231 \\
\hline Mean systolic BP over 5 years (mmHg) & $132.5 \pm 10.1$ & $134.9 \pm 12.2$ & 0.259 \\
\hline Diastolic BP at the baseline ( $\mathrm{mmHg}$ ) & $70.3 \pm 9.3$ & $71.8 \pm 9.7$ & 0.433 \\
\hline Mean diastolic BP over 5 years $(\mathrm{mmHg})$ & $71.8 \pm 6.5$ & $72.4 \pm 8.7$ & 0.734 \\
\hline Mean PD at the baseline (mm) & $2.0 \pm 0.4$ & $2.1 \pm 0.3$ & 0.287 \\
\hline Mean CAL at the baseline (mm) & $2.9 \pm 0.7$ & $3.1 \pm 0.7$ & 0.138 \\
\hline HDL level at the baseline (mg/dL) & $60.3 \pm 15.6$ & $59.8 \pm 21.5$ & 0.893 \\
\hline LDL level at the baseline (mg/dL) & $119.7 \pm 25.6$ & $104.1 \pm 26.8$ & 0.005 \\
\hline \multirow[t]{2}{*}{$\mathrm{TC}$ level at the baseline $(\mathrm{mg} / \mathrm{dL})$} & $206.2 \pm 28.8$ & $194.1 \pm 28.5$ & 0.044 \\
\hline & \multicolumn{2}{|c|}{ N (\%) } & $p$-value ${ }^{\mathrm{b}}$ \\
\hline \multicolumn{4}{|l|}{ Gender } \\
\hline Male & $36(50.0)$ & $21(60.0)$ & 0.331 \\
\hline Female & $36(50.0)$ & $14(40.0)$ & \\
\hline \multicolumn{4}{|l|}{ Periodontal condition at the baseline } \\
\hline Healthy & $51(70.8)$ & $14(40.0)$ & 0.002 \\
\hline Poor condition $^{\mathrm{c}}$ & $21(29.2)$ & $21(60.0)$ & \\
\hline \multicolumn{4}{|l|}{ Lipid condition at the baseline } \\
\hline Favorable & $46(63.9)$ & $22(62.9)$ & 0.917 \\
\hline Unfavorable $^{\mathrm{d}}$ & $26(36.1)$ & $13(37.1)$ & \\
\hline \multicolumn{4}{|l|}{ Smoking status ${ }^{\mathrm{e}}$} \\
\hline Non-smoker & $64(88.9)$ & $30(85.7)$ & 0.921 \\
\hline Smoker & $8(11.1)$ & $4(11.4)$ & \\
\hline
\end{tabular}

BMI; Body mass index, BP; Blood pressure, PD; Probing depth, CAL; Clinical attachment level, HDL; High density lipoprotein, LDL; Low density lipoprotein, TC; Total cholesterol; ${ }^{a} t$-test; ${ }^{b}$ Chi-square test; ${ }^{\circ}$ Mean PD $\geq 2 \mathrm{~mm}$ and mean CAL $\geq 2.5 \mathrm{~mm}$; ${ }^{\mathrm{d}}$ Serum LDL-C level $\geq 140 \mathrm{mg} / \mathrm{dL}$ and/or serum HDL-C level $<40$ $\mathrm{mg} / \mathrm{dL}$ and/or serum TC $\geq 220 \mathrm{mg} / \mathrm{dL}$; ${ }^{\mathrm{e}}$ Data was missing for 1 subject.

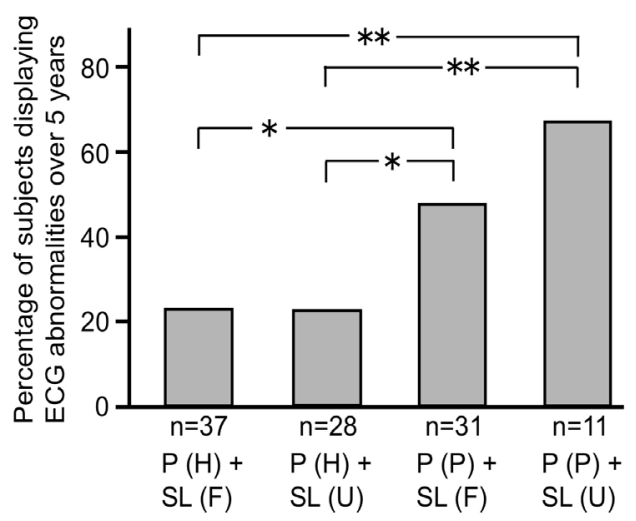

Figure 1. Percentage of subjects that displayed ECG abnormalities over 5 years in four groups according to their periodontal condition and serum lipid level [P (H); Healthy periodontal condition, $\mathrm{P}(\mathrm{P})$; Poor periodontal condition, SL (F); Favorable serum lipid profile, SL (U); Unfavorable serum lipid profile]. ${ }^{*} p<0.05,{ }^{*} p<0.01$. compared with the subjects who exhibited a healthy periodontal condition and a favorable serum lipid profile $(21.6 \%, p=0.008)$, or a healthy periodontal condition and an unfavorable serum lipid profile, at the baseline $(21.4 \%, p=0.010)$. Furthermore, the frequency of ECG abnormalities was significantly lower in the latter two groups than in the other two groups $(21.5 \%$ vs $50.0 \%, p$ $=0.002$ ).

The results of the multivariate logistic regression analysis are shown in Table 2. The subjects who displayed a poor periodontal condition at the baseline were found to be at a 2.97 times higher risk of exhibiting ECG abnormalities than the subjects who displayed a healthy periodontal condition at the baseline $(p=0.019)$. Moreover, BMI at the baseline was positively associated with the presence of ECG abnormalities $(\mathrm{OR}=1.22, p=0.019)$ while the LDL-C level at the baseline had a significant negative effect on the risk of ECG abnormalities (OR = $0.96, p=0.019)$. 
Table 2. Binary logistic regression analysis of the effects of the explanatory variables including periodontal condition on the presence of ECG abnormalities.

\begin{tabular}{cccccc}
\hline \multirow{2}{*}{ Independent variable } & \multicolumn{4}{c}{ Dependent variable: Presence of ECG abnormalities over 5 years } \\
\cline { 2 - 6 } & Coefficient & SE & $\boldsymbol{p}$-value & OR & $\mathbf{9 5 \%}$ CI \\
\hline Periodontal condition (0: healthy/1: poor) & 1.09 & 0.47 & $\mathbf{0 . 0 1 9}$ & $\mathbf{2 . 9 7}$ & $1.19-7.38$ \\
TC at the baseline & 0.02 & 0.02 & 0.227 & 1.02 & $0.99-1.05$ \\
LDL at the baseline & -0.04 & 0.02 & $\mathbf{0 . 0 1 9}$ & $\mathbf{0 . 9 6}$ & $0.92-0.99$ \\
BMI at the baseline & 0.20 & 0.09 & $\mathbf{0 . 0 1 9}$ & $\mathbf{1 . 2 2}$ & $1.03-1.45$ \\
\hline
\end{tabular}

TC; Total cholesterol, LDL; Low density lipoprotein, BMI; Body mass index, SE; Standard error, OR; Odds ratio, CI; Confidence interval.

\section{DISCUSSION}

An association between periodontal condition and the presence of ECG abnormalities was detected in this study. It has been suggested that dental caries and periodontal disease are related to myocardial infarction [20], dysrhythmia [21], coronary heart disease [4], and abnormal ECG findings in octogenarians [17]. Furthermore, some studies have reported that periodontitis results in higher systemic levels of C-reactive protein and interleukin-6 and increased numbers of neutrophils, suggesting that elevated levels of these inflammatory substances cause inflammatory changes in atherosclerotic lesions, increasing the risk of cardiac or cerebrovascular disease events $[22,23]$. Other recent studies have suggested that $S$. mutans migrates to cardiovascular endothelial tissue [24] and binds to extracellular matrix molecules and fibrinogen [25]. Moreover, DNA from periodontal pathogens was highly prevalent in coronary artery atherosclerosis samples taken from patients with coronary artery disease [26]. These findings support the view that infection with oral bacterial species could be a risk factor for coronary heart disease.

A negative association between the serum LDL-C level and the presence of ECG abnormalities during the study period was found in this study. One possible explanation for this inverse association is the effect of selective survival; i.e., those who are susceptible to the biological effects of high cholesterol levels tend to die before reaching an advanced age $[27,28]$. Thus, the individuals who lived longer would form an unintentionally selected group with lower cholesterol levels and a genetic background (or other factors) that protects them from the effects of higher cholesterol concentrations [29]. This explanation agrees with the fact that the subjects in the present study were relatively healthy and did not require special assistance for their daily activities. Another study stated that in the very elderly, changes in the vessel wall might lower their susceptibility to cholesterol [28].

This finding might also explain our study results. The subjects who exhibited a poor periodontal condition and unfavorable serum lipid levels at the baseline displayed the highest frequency of ECG abnormalities during the study period. The difference in the frequency of ECG abnormalities was greatest between the subjects who did and did not display a healthy periodontal condition, regardless of their baseline serum lipid levels. Moreover, no significant difference in the frequency of ECG abnormalities was found between the subjects with and without favorable serum lipid levels who displayed the same periodontal condition. Therefore, periodontal condition was more closely associated with ECG abnormalities than serum lipid levels at the baseline. One possible explanation for this is that the subjects were relatively healthy and independent community-dwelling elderly individuals who had few problems with their general health, particularly with their serum lipid profiles. Another possible explanation is that we did not include serum triglycerides when assessing serum lipid condition since non-fasting blood samples were collected. Since serum triglycerides might influence a patient's lipid profile, this should be considered as one of the limitations of our study. Another possible limitation of our study was the fact that we did not possess information about the medication being taken by the subjects, which might have also had an influence on the presence/absence of ECG abnormalities, nor did we have any information about the other medical conditions suffered by the subjects, such as their blood glucose levels, which could have affected their systemic condition.

\section{CONCLUSION}

Our results suggest that in the elderly periodontal condition has a significantly positive effect on the risk of ECG abnormalities, while the LDL-C level has the opposite effect. These findings indicate that periodontal condition could be used as a marker of coronary heart disease, even in the healthy elderly. Therefore, it is important to recognize periodontitis as a possible marker for coronary heart disease with an inverse association of serum lipid condition in the elderly.

\section{ACKNOWLEDGEMENTS}

The authors declare that they have no conflict of interests. This study 
was supported by a grant-in-aid from the Ministry of Health and Welfare of Japan (H10-Iryo-001). We are profoundly grateful to the study participants for their assistance.

\section{REFERENCES}

[1] Petersen, P.E., Bourgeois, D., Ogawa, H., Estupinan-Day, S. and Ndiaye, C. (2005) The global burden of oral diseases and risks to oral health. Bulletin of the World Health Organization, 83, 661-669.

[2] Papapanou, P.N. (1999) Epidemiology of periodontal diseases: An update. Journal of the International Academy of Periodontology, 1, 110-116.

[3] Petersen, P.E. (2006) Oral health-general health interrelationships: Health policy implications. Inside Dentistry, 2, $1-5$.

[4] DeStefano, F., Anda, R.F., Kahn, H.S., Williamson, D.F. and Russell, C.M. (1993) Dental disease and risk of coronary heart disease and mortality. British Medical Journal, 306, 688-691.

http://dx.doi.org/10.1136/bmj.306.6879.688

[5] Corti, M.C., Guralnik, J.M., Salive, M.E., Harris, T., Field, T.S., et al. (1995) HDL cholesterol predicts coronary heart disease mortality in older persons. Journal of American Medical Association, 274, 539-544. http://dx.doi.org/10.1001/jama.1995.03530070037026

[6] Izumi, A., Yoshihara, A., Hirotomi, T. and Miyazaki, H. (2009) The relationship between serum lipids and periodontitis in elderly non-smokers. Journal of Periodontology, 80, 740-748. http://dx.doi.org/10.1902/jop.2009.080584

[7] Saito, T., Shimazaki, Y. and Sakamoto, M. (1998) Obesity and periodontitis. The New England Journal of Medicine, 339, 482-483. http://dx.doi.org/10.1056/NEJM199808133390717

[8] Wakai, K., Kawamura, T., Umemura, O., Hara, Y., Machida, J., et al. (1999) Associations of medical status and physical fitness with periodontal disease. Journal of Clinical Periodontology, 26, 664-672. http://dx.doi.org/10.1034/j.1600-051X.1999.261006.x

[9] Holm-Pedersen, P., Avlund, K., Morse, D.E., Stoltze, K., Katz, R.V., et al. (2005) Dental caries, periodontal disease, and cardiac arrhythmias in community-dwelling older persons aged 80 and older: Is there a link? Journal of American Geriatric Society, 53, 430-437. http://dx.doi.org/10.1111/j.1532-5415.2005.53160.x

[10] Sridhar, R., Byakod, G., Pudakalkatti, P. and Patil, R. (2009) A study to evaluate the relationship between periodontitis, cardiovascular disease and serum lipid levels. International Journal of Dental Hygiene, 7, 144-150. http://dx.doi.org/10.1111/j.1601-5037.2008.00318.x

[11] Schulz, K.F. and Grimes, D.A. (2002) Case-control studies: Research in reverse. Lancet, 359, 431-434. http://dx.doi.org/10.1016/S0140-6736(02)07605-5

[12] Saxlin, T., Suominen-Taipale, L., Kattainen, A., Marniemi, J., Knuuttila, M., et al. (2008) Association between serum lipid levels and periodontal infection. Journal of Clinical Periodontology, 35, 1040-1047.
http://dx.doi.org/10.1111/j.1600-051X.2008.01331.x

[13] Rich, M.W. (2009) Epidemiology of atrial fibrillation. Journal of Interventional Cardiac Electrophysiology, 25, 3-8. http://dx.doi.org/10.1007/s10840-008-9337-8

[14] Albandar, J.M., Brunelle, J.A. and Kingman, A. (1999) Destructive periodontal disease in adults 30 years of age and older in the United States, 1988-1994. Journal of Periodontology, 70, 13-29. http://dx.doi.org/10.1902/jop.1999.70.1.13

[15] Shimazaki. Y., Saito, T., Kiyohara, Y., Kato, I., Kubo, M., et al. (2004) Relationship between electrocardigraphic abnormalities and periodontal disease: The Hisayama study. Journal of Periodontology, 75, 791-797. http://dx.doi.org/10.1902/jop.2004.75.6.791

[16] Rautuharju, P.M., Park, L., Rautuharju, F.S. and Crow, R. (1998) A standardized procedure for locating and documenting ECG chest electrode positions, consideration of the effect of breast tissue on ECG amplitudes in women. Journal of Electrocardiology, 31, 17-29. http://dx.doi.org/10.1016/S0022-0736(98)90003-6

[17] Takata, Y., Ansai, T., Matsumura, K., Awano, S., Hamasaki, T., et al. (2001) Relationship between tooth loss and electrocardiographic abnormalities in octogenarians. Journal of Dental Research, 80, 1648-1652. http://dx.doi.org/10.1177/00220345010800071001

[18] Ichihara, Y., Sugino, M., Hattori, R., Anno, T., Mizuno, Y., et al. (1997) Relation of electrocardiographic left ventricular hypertrophy with and without T-wave changes to systemic blood pressure, body mass, and serum lipid and blood glucose level in Japanese men. The American Journal of Cardiology, 80, 730-735. http://dx.doi.org/10.1016/S0002-9149(97)00504-3

[19] Teramoto, T., Sasaki, J., Ueshima, H., Egusa, G., Kinoshita, M., et al. (2007) Executive summary of Japanese Atherosclerosis Society (JAS) guideline for diagnosis and prevention of atherosclerotic cardiovascular diseases for Japanese. Journal of Atherosclerosis and Thrombosis, 14, 45-50. http://dx.doi.org/10.5551/jat.14.45

[20] Mattila, K.J., Nieminen, M.S., Valtonen, V.V., Rasi, V.P., Kesaniemi, A., et al. (1989) Association between dental health and acute myocardial infarction. British Medical Journal, 298, 779-781. http://dx.doi.org/10.1136/bmj.298.6676.779

[21] Kaneko M, Yoshihara A and Miyazaki H. (2011) Relationship between root caries and cardiac dysrhythmia. Gerodontology, 28, 289-295. http://dx.doi.org/10.1111/j.1741-2358.2010.00367.x

[22] Saito, T., Murakami, M., Shimazaki, Y. Oobayashi, K., Matsumoto, S., et al. (2003) Association between alveolar bone loss and elevated serum C-Reactive Protein in Japanese men. Journal of Periodontology, 74, 1741-1746. http://dx.doi.org/10.1902/jop.2003.74.12.1741

[23] Loos, B.G., Craandijk, J., Hoek, F.J., Wertheim van Dillen, P.M.E. and van der Velden, U. (2000) Elevation of systemic markers related to cardiovascular diseases in the peripheral blood of periodontitis patients. Journal of Periodontology, 71, 1528-1534. http://dx.doi.org/10.1902/jop.2000.71.10.1528

[24] Nakano, K., Nemoto, H., Nomura, R., Homma, H., Yo- 
shioka, H., et al. (2006) Serotype distribution of Streptococcus mutans a pathogen of dental caries in cardiovascular specimens from Japanese patients. Journal of Medical Microbiology, 56, 551-556.

http://dx.doi.org/10.1099/jmm.0.47051-0

[25] Beg, A.M., Jones, M.N., Miller-Torbert, T. and Holt R.G. (2002) Binding of Streptococcus mutans to extracellular matrix molecules and fibrinogen. Biochemical and Biophysical Research Communications, 298, 75-79. http://dx.doi.org/10.1016/S0006-291X(02)02390-2

[26] Pucar, A., Milasin, J., Lekovic, V., Vulkadinovic, M., Ristic, M., et al. (2007) Correlation between atherosclerosis and periodontal putative pathogenic bacterial infections in coronary and internal mammary arteries. Journal of Periodontology, 78, 677-682. http://dx.doi.org/10.1902/jop.2007.060062
[27] Brescianini, S., Maggi, S., Farchi, G., Mariotti, S., Carlo, A.D., et al. (2003) Low total cholesterol and increased risk of dying: are low levels clinical warning signs in the elderly? Results from the Italian Longitudinal Study on Aging. Journal of American Geriatric Society, 51, 991996. http://dx.doi.org/10.1046/j.1365-2389.2003.51313.x

[28] Weverling-Rijnsburger, A.W., Blauw, G.J., Lagaay, A.M., Knook, D.L., Meinders, A.E., et al. (1997) Total cholesterol and risk of mortality in the oldest old. Lancet, 350, 1119-1123. http://dx.doi.org/10.1016/S0140-6736(97)04430-9

[29] Schatz, I.J., Masaki, K., Yano, K., Chen, R., Rodrigues, B.L. et al. (2001) Cholesterol and all-cause mortality in elderly people from the Honolulu heart program: A cohort study. Lancet, 358, 351-355. http://dx.doi.org/10.1016/S0140-6736(01)05553-2 